\title{
Characteristics of Forbush decreases measured by means of the new scintillation muon hodoscope $\mathrm{ScMH}$
}

\author{
I.I. Yashin 1, N.V. Ampilogov, I.I. Astapov, N.S. Barbashina, A.N. Dmitrieva, \\ K.G. Kompaniets, A.A. Kovylyaeva, V.V. Shutenko \\ National Research Nuclear University MEPhI (Moscow Engineering Physics Institute) \\ Kashirskoe shosse 31, Moscow, 115409, Russia \\ E-mail:IIYashin@mephi.ru
}

\begin{abstract}
The paper presents results of investigation of characteristics of Forbush decreases that occurred in 2016 and were detected by muon hodoscope ScMH. For this, barometric coefficients and differential temperature coefficients (DTC) were calculated, allowing to make corrections in the counting rate with considering of the temperature changes at all altitudes of the atmosphere. Obtained characteristics were compared with results of registration of same events with the URAGAN muon hodoscope. Comparison of results obtained with both detectors demonstrated a good agreement.
\end{abstract}




\section{Introduction}

Passing through the heliosphere, primary cosmic ray flux is modulated by processes related with the solar activity, that lead to muon flux variations on the Earth surface. Also, the intensity of the muon flux at the surface is sensitive to different thermodynamic atmospheric processes. Thus, measuring of the muon flux intensity gives us a possibility to investigate different phenomena in heliosphere, magnetosphere and atmosphere of the Earth. The method which provides this way of remote monitoring is named a muon diagnostics [1]. One of the tasks of the muon diagnostics of processes in heliosphere and Earth's magnitosphere is the analysis of Forbush decreases (FD). FD is the sharp decrease of the flux of primary cosmic rays in the heliosphere caused by disturbances of the interplanetary magnetic field caused by the solar activity. For implementation of the method of muon diagnostics for investigation of FD, the coordinate-tracking detectors of muons - hodoscopes - are necessary. The appearance of multidirectional muon telescopes and later of muon hodoscopes revealed new possibilities in studies of Forbush decreases [2]. Taking into account the experience of the muon hodoscope URAGAN design and operation [3], a new scintillation muon hodoscope ScMH was created [4]. The important advantage of muon hodoscope is a possibility of simultaneous registration of muons from different directions and to form "muon images" of the sky hemisphere. In this work, the analysis of the Forbush decreases registered by ScMH and URAGAN in 2016 is considered.

\section{Detectors}

Muon hodoscope URAGAN [1] and scintillation muon hodoscope ScMH are operated as a part of the Unique Scientific Facility "Experimental complex NEVOD". The URAGAN supermodule includes eight planes interlaid with $5 \mathrm{~cm}$ foam plastic, each composed of 320 streamer tubes with external strips (along and across streamer tubes) forming two-dimensional readout system (608 data channels in each plane). Total area of one supermodule is about 11.5 $\mathrm{m}^{2}$. The setup provides detection of particles in a wide range of zenith angles (from 0 to $80^{\circ}$ ) with angular accuracy about $0.7^{\circ}$. The data processing system allows to reconstruct muon tracks in on-line mode and to register muon flux from the upper hemisphere as a continuous sequence of 2D matrices. Threshold energies of the URAGAN depend on zenith angle and lie within limits from $200 \mathrm{MeV}$ to $450 \mathrm{MeV}$.

The scintillation muon hodoscope is a coordinate-tracking muon detector which consists of two identical independent detectors - supermodules (SM1 and SM2). Each SM has a sensitive area about $11.6 \mathrm{~m}^{2}$, a good spatial (about $2.5 \mathrm{~cm}$ ) and angular (better than $2^{\circ}$ ) accuracy, a wide zenith angle aperture of muon registration $0^{\circ}-75^{\circ}$. A long narrow strip (with dimensions $10.6 \mathrm{~mm} \times 26.3 \mathrm{~mm} \times 3460 \mathrm{~mm}$ ) of plastic scintillator with wavelength shifting (WLS) fiber is a basic detection element of ScMH $[4,5]$. SM consists of four $\mathrm{X}-\mathrm{Y}$ coordinate planes (CP) mounted in a common assembly. Each CP consists of two coordinate layers with orthogonally oriented strips, which provide measurements of $\mathrm{X}$ and $\mathrm{Y}$ coordinates of muon track crossing point. Coordinate layer consists of two adjacent modules - basic non-dismountable modules $(\mathrm{BM})$ of the detector, with 64 scintillation strips in a common housing. BM has a single multi- 
anode photomultiplier to registration of light pulses from all 64 strips. One end of each WLS fiber is positioned opposite the corresponding cell of 64-channel multi-anode photomultiplier.

\section{The analysis of Forbush decreases in data of scintillation muon hodoscope ScMH and muon hodoscope URAGAN}

For the analysis of Forbush decreases, the 10-minute average counting rates of muon detectors ScMH and URAGAN were used. Corrections for the barometric and temperature effect were introduced. During the operation of muon hodoscopes in 2016, four Forbush decreases were detected. Figure 1 shows counting rates of scintillation muon hodoscope and muon hodoscope URAGAN during FD February 12, 2016.

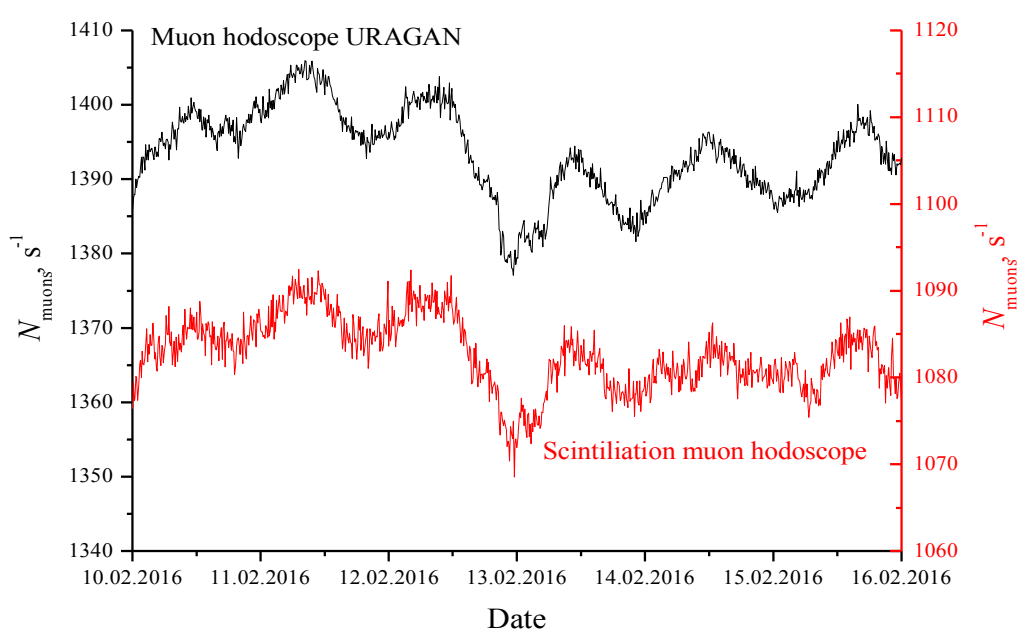

Figure 1. The average counting rates of the muon hodoscope URAGAN (left axis) and the scintillation muon hodoscope (right axis), calculated at 10-minute intervals of the data sets, during FD from February $12,2016$.

Amplitude of cosmic rays flux decreasing is one of the main parameters of the Forbush effect. Therefore it is important to measure it correctly and with low uncertainties. The difficulties are related with diurnal cycle and various trends, which modulate behavior of cosmic rays intensity. This problem is solved using averaging of muon counting rates before and after Forbush decrease over several intervals (one, two, three days) and determination of FD amplitude as a difference between averaged values. Depending on variants of averaging, several values of FD amplitude were obtained. The difference between them represents the systematic uncertainty connected with diurnal cycles changing. The amplitude of a given Forbush decrease $\left(A_{\mathrm{FD}}\right)$ is defined as the average value among a set of different amplitude estimate variants, and its errors are defined as rms deviations over the set. The amplitudes of four FD registered by means of muon hodoscopes and calculated using the described method are presented in Table 1.

Table 1: Amplitudes of Forbush decreases in muon counting rate.

\begin{tabular}{|c|c|c|c|}
\hline FD & URAGAN, \% & ScMH SM1, \% & ScMH SM2, \% \\
\hline 9 February 2016 & $0.97 \pm 0.02$ & $0.83 \pm 0.03$ & - \\
\hline 12 February 2016 & $1.14 \pm 0.01$ & $0.98 \pm 0.01$ & - \\
\hline 11 March 2016 & $1.01 \pm 0.02$ & $0.79 \pm 0.01$ & $0.93 \pm 0.02$ \\
\hline 07 July 2016 & $1.04 \pm 0.01$ & $0.77 \pm 0.02$ & $0.86 \pm 0.01$ \\
\hline
\end{tabular}




\section{Methods and results of the analysis of FD according to the muon flux spatial-angular characteristics}

Figure 2 shows an example of FD occured on February 12, 2016. It can be clearly seen in the variations of the counting rates of muon hodoscopes ScMH and URAGAN. In Figure 2, also parameters of the interplanetary magnetic field (IMF, the total induction of the interplanetary magnetic field $B_{t}$ and the third component of IMF that is perpendicular to the ecliptic, $B_{z}$ ), and the solar wind velocity $\left(V_{\mathrm{SW}}\right)$ are shown. The coronal mass ejection $(\mathrm{CME})$ with a maximum speed $977 \mathrm{~km} / \mathrm{s}$ and with average velocity up to $656 \mathrm{~km} / \mathrm{s}$ at 13:36 February 11 was observed. Than at 21:28 on the same day a second CME (halo II type) with an average speed of up to 637 $\mathrm{km} / \mathrm{s}$ was observed. Most likely that the variations in the counting rates of muon hodoscopes ScMH and URAGAN was associated with the first CME.

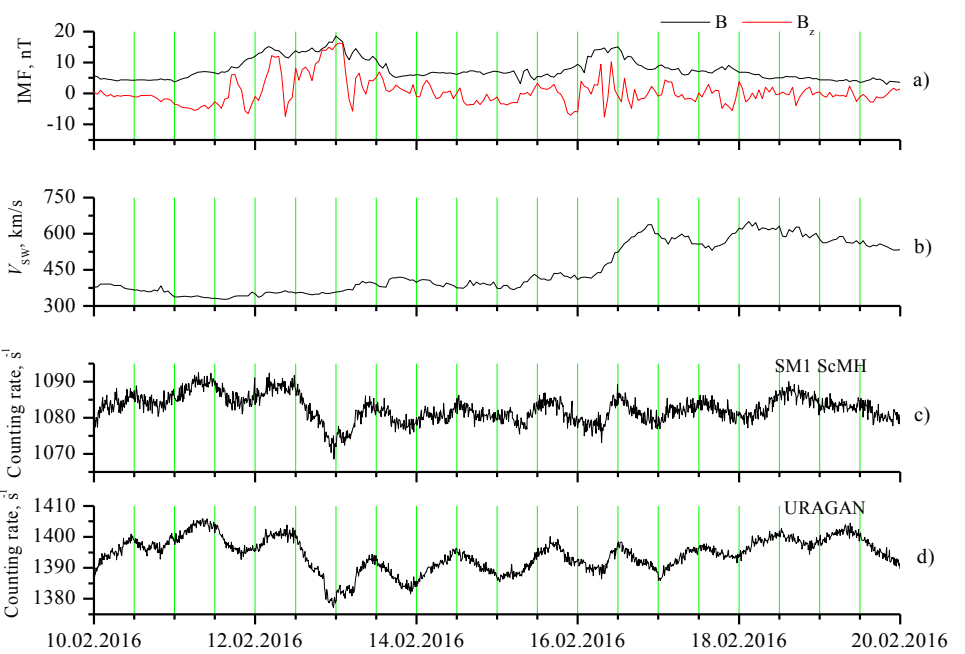

Figure 2: a) Induction of the interplanetary magnetic field $B_{t}$ and $B_{z}$ component; b) solar wind velocity; c) SM1 ScMH counting rate, d) URAGAN counting rate.

According to the ACE satelite [6], the induction of the interplanetary magnetic field began to increase on February 11 at 14:00. The IMF parameters $B_{t}$ and $B_{z}$ changed to 19 and $-8 \mathrm{nT}$, correspondingly. An increase in the speed of the solar wind was not observed. Geomagnetic disturbances were registered on the Earth: the maximum of $D_{\text {st }}$-index on February 12 at 10:00 and the increase in the $K_{p}$-index to the level 4 from February 11 at 21:00 to February 11 at 06:00 and from February 12 at 09:00 to February 12 at 12:00.

Muon hodoscope response represents the information about triggered strips in each of the two projection planes XZ and YZ. Track parameters (two projection angles) are reconstructed in each projection plane in a real time mode and are accumulated in two-dimensional array $\left(\theta_{\mathrm{X}}, \theta_{\mathrm{Y}}\right)$ during one-minute interval. Each matrix is normalized to the average matrix obtained by averaging minute matrices for the preceding 24 hours [1]. The result is a matrix, each cell of which contains deviations from the average one in units of their statistical errors. To smooth sharp fluctuation peaks in separate bins, all values of the matrix are smoothed using the Gaussian filter. To optimize the presentation of information considering low statistics in the matrix corners, it is reasonable to present it in the form of a circle limited by zenith angle of $75^{\circ}$. 
Such data array (matrix) represents a "muon snapshot" (muonograph) of the upper hemisphere limited by the detector aperture. For four FD, the muon snapshots that give visual information about the variations of the muon flux angular distribution during the event were plotted. Figure 3 and 4 show the one-hour exposure muon snapshots of SM1 ScMH and of muon hodoscope URAGAN during the FD of February 12, 2016. Each row of the snapshots in the figure corresponds to the different phase of the FD development: the first row reflects the period before the beginning of the Forbush decrease (11/02/2016 22:00 - 12/02/2016 16:00); the second, phase of the decrease (12/02/2016 17:00 - 13/02/2016 01:00); then, phase of the minimum (13/02/2016 02:00 - 13/02/2016 06:00); the last row corresponds to the phase of recovery $(13 / 02 / 201608: 00-13 / 02 / 2016$ 13:00).

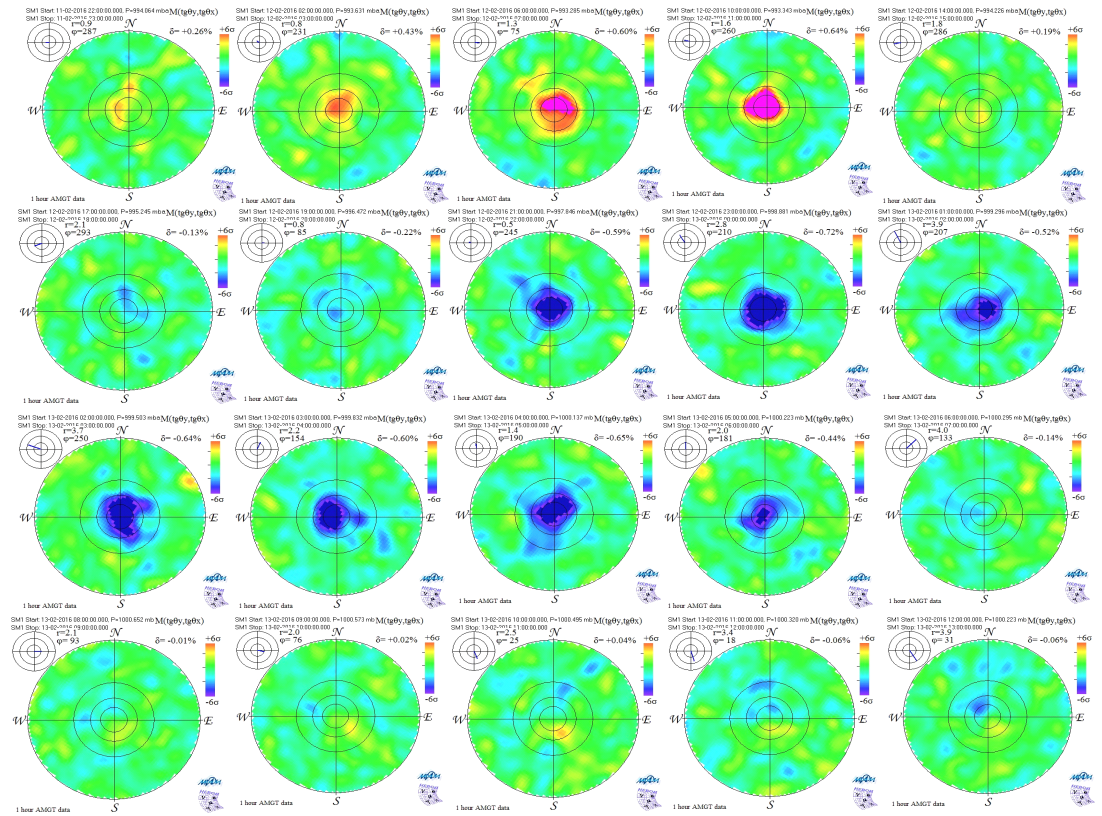

Figure 3: Muon snapshots of SM1 ScMH during the FD of February 12, 2016.

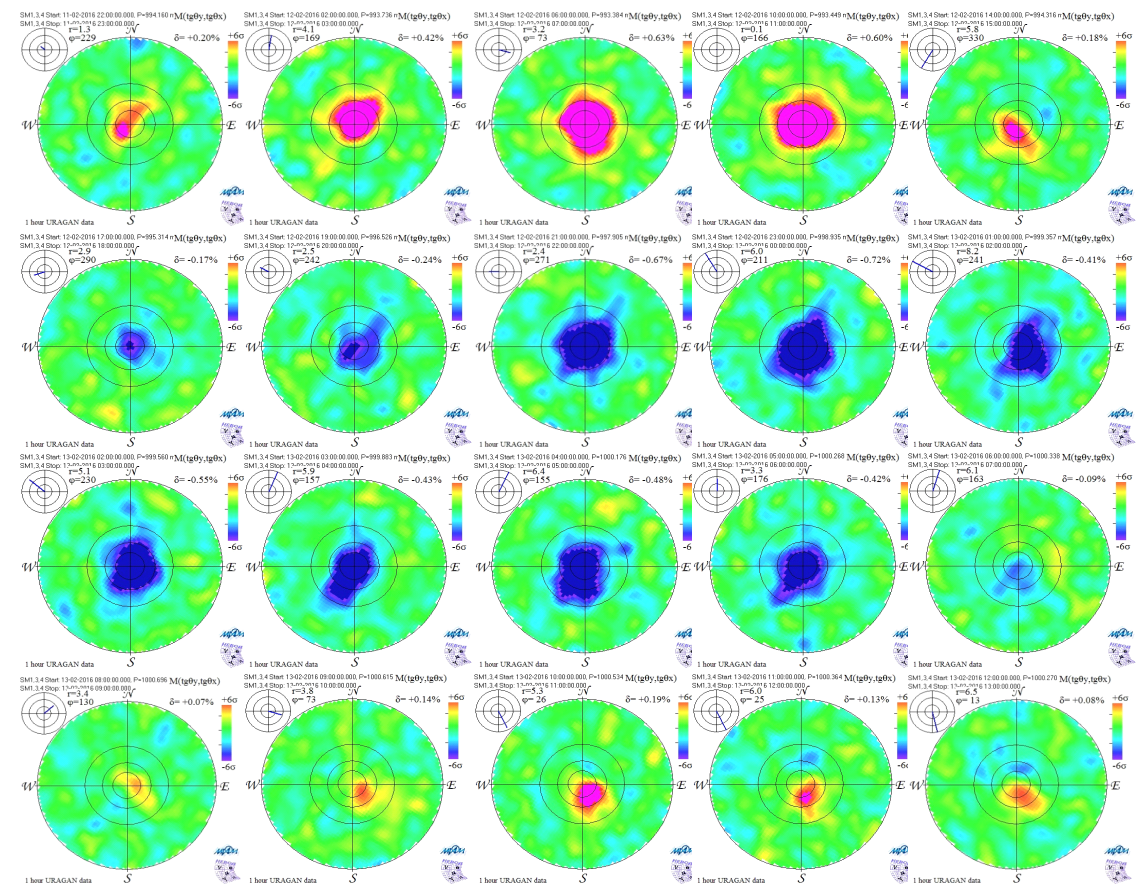

Figure 4: Muon snapshots of muon hodoscope URAGAN during the FD of February 12, 2016 


\section{Conclusion}

New scintillation muon hodoscope operating in the experimental complex NEVOD gives a possibility to investigate Forbush decreases in muon flux. Analysis of data from the ScMH allows to study characteristics and muons snapshots of Forbush effects. The obtained characteristics were compared with results of registration of the same events with the help of the URAGAN muon hodoscope. Comparison of results obtained with both detectors demonstrated a good agreement.

This work was performed at the Unique Scientific Facility "Experimental complex NEVOD" with the financial support of the MEPhI Academic Excellence Project (agreement with the Ministry of Education and Science of the Russian Federation of August 27, 2013, project no. 02.a03.21.0005).

\section{References}

[1] I.I. Yashin et al., Advances in Space Research, 56, 12 (2015).

[2] A.V. Belov et al., Bull. Russ. Acad. Sci.: Phys., 65, 373 (2001), in Russian.

[3] N.S. Barbashina et al., Instrum. Experim. Techn., 51, 180 (2008).

[4] N.V. Ampilogov et al., Astrophys. Space Sci. Trans., 7, 435 (2011).

[5] N.V. Ampilogov et al., Journal of Physics: Conference Series, 3, 675 (2016).

[6] OMNI Database: ftp://nssdcftp.gsfc.nasa.gov/spacecraft_data/omni 